\title{
MÉTODOS PARA CAPTURA DE LIÇÕES APRENDIDAS: EM DIREÇÃO A MELHORIA CONTINUA NA GESTÃO DE PROJETOS
}

\section{RESUMO}

Em um mercado competitivo e em constante evolução a gestão efetiva da informação e a transferência de conhecimento são aspectos estratégicos para as empresas. A fim de melhorar os processos de gestão de projetos, as empresas têm adotado metodologias e frameworks, como alternativas que permitam maximizar a taxa de sucesso no desenvolvimento dos projetos. Como uma extensão da gestão de projetos, o processo de captura e análise de lições aprendidas é um método eficiente e efetivo de gestão da informação e transferência de conhecimento, adquiridos durante a execução de um projeto, permitindo que a organização aprimore seus processos, reduza custos e gere valor ao negócio. Cada projeto executado fornece uma base de conhecimento importante que pode ser utilizada como diferencial competitivo pela empresa. Este artigo teórico apresenta uma revisita e análise dos principais métodos para captura de lições aprendidas em projetos propostos nas últimas décadas. A análise foi realizada a partir de critérios comuns a maior parte dos métodos. Os resultados incluem recomendações para promover o aprendizado sistemático em projetos e a implementação exitosa da gestão de lições aprendidas.

Palavras-chave: Lições Aprendidas; Gestão de Projetos; Aprendizagem em Projetos; Aprendizado Sistemático.

\begin{abstract}
In a competitive market which is in constant evolution, the effective information management and the knowledge transfer are strategic aspects to the organizations. In order to improve their project management processes, companies have adopted methodologies and frameworks as alternatives to maximize the success index in their projects. As an extension of project management, the process of capture and analysis of lessons learned is an efficient and effective method to manage information and to enable the knowledge transfer, improving their process, reducing costs and aggregating value. Each executed project provides a valuable knowledge base to be used as a competitive differential. This theorical paper presents a review and analysis in the mainly methods of lessons learned capture proposed in the last decades. The analysis was performed on the basis of common criteria. The results include a set of recommendations to promote the systematic learning in projects and the success implementation of lessons learned management.
\end{abstract}

Keywords: Lessons Learned; Project Management; Project Learning; Systematic Learning.

Giuliana Santos Veronese ${ }^{1}$

\footnotetext{
${ }^{1}$ Doutora em Engenharia em Informática pala Universidade de Lisboa - UL, Portugal. Executiva em ambiente corporativo gerenciando equipes através da inovação e criatividade. Brasil. E-mail: santos.giuliana@gmail.com
} 


\section{INTRODUÇÃO}

Os padrões da economia atual exigem das organizações uma atenção especial na melhoria contínua dos seus processos. Empresas têm buscado métodos e estratégias a fim de garantir o diferencial de seus produtos e serviços gerando vantagem competitiva frente aos seus concorrentes. A fim de melhorar os processos de gestão de projetos, as empresas têm adotado metodologias e frameworks, como alternativas que permitam maximizar a taxa de sucesso no desenvolvimento dos projetos.

Como uma extensão da gestão de projetos, o processo de captura e análise de lições aprendidas é um método eficiente e efetivo de transferência de conhecimento adquirido durante a execução de um projeto, permitindo que a organização aprimore seus processos, reduza custos e gere valor ao negócio. Cada projeto executado fornece uma base de conhecimento importante que pode ser utilizada como diferencial competitivo pela empresa.

Entretanto, o uso de lições aprendidas prevê o estudo e a implantação de uma metodologia de transferência de conhecimento e não se limita apenas a um conjunto de atividades executadas durante uma determinada fase do projeto.

Um dos principais desafios que as organizações enfrentam é o pouco incentivo para implantação de um processo estruturado de lições aprendidas (Hobday, 2000). Embora este processo permita as empresas melhorar as competências na gestão de projetos, organizações tem falhado em promover um ambiente para a utilização correta das lições aprendidas. Uma das principais dificuldades dos gerentes de projetos é administrar as lições aprendidas. Estudos mostram que apesar de $62.4 \%$ das organizações têm definidos procedimentos para a documentação das lições aprendidas, entretanto, $89,3 \%$ das organizações não seguem estes procedimentos (Williams, 2008).

Os benefícios de uma gestão adequada das lições aprendidas são inquestionáveis, por outro lado o uso equivocado pode acarretar em aumento dos custos dos projetos, horários estendidos, falta de comunicação, retrabalho e repetição de erros (Jugdev, 2012). Esses problemas geralmente são ocasionados pela falta de definição e suporte de como documentar as lições aprendidas gerando uma documentação superficial que não permite o reuso e/ou favorece a utilização equivocada da informação. Para ultrapassar este tipo de dificuldade, todos os envolvidos na aplicação dos métodos de captura e análise de lições aprendidas devem conhecer seus principais aspectos a fim de identificar quais desses métodos melhor se aplicam a realidade específica da organização e a natureza de seus projetos.

Uma evidência da relevância das lições aprendidas tanto para as organizações quanto para os projetos é o fato deste tema ser recorrente na literatura em geral (Senge, 1991; Collier, DeMarco, \& Fearey, 1996; Busby, 1999; Weber, Aha, \& Becerra-Fernandez, 2001; Williams, 2008: Baaz, Holmberg, Nilsson, Olsson, \& Sandberg, 2010; Swan, Scarbrough, \& Newell, 2010; Cheah, Khoh, \& Ooi, 2011) e, especificamente em gestão de projetos (Loo, 2002; Schindler \& Eppler, 2003; Sense, 2004; Anbari, Carayannis, \& Voetsch, 2008; Sense, 2008; Jugdev, 2012; Ferenhof, Forcellini, \& Varvakis, 2013).

Este artigo teórico apresenta um estudo sobre os principais aspectos dos métodos de captura e análise de lições aprendidas mais discutidos e referenciados na literatura. As principais contribuições deste artigo tanto para a literatura em gestão de projetos quanto para aprendizagem organizacional são:

- Revisita e análise dos métodos para captura de lições aprendidas adequados para serem usados durante o ciclo de vida da gestão de projetos;

- Uma proposta de recomendações para promover o aprendizado em projetos por meio da gestão de lições aprendidas.

Este artigo está organizado como segue: a seção 2 apresenta os principais métodos para captura de lições aprendidas encontrados numa literatura interdisciplinar. A seção 3 analisa os métodos de captura de lições aprendidas no contexto da gestão de projetos. A seção 4 apresenta uma discussão sobre a análise realizada. A seção 5 conclui o artigo e faz recomendações de aplicação dos métodos de captura de lições aprendidas no ciclo de vida da gestão de projetos.

\section{MÉTODOS E ATIVIDADES PARA CAPTURA DE LIÇÕES APRENDIDAS}

A literatura apresenta diversos métodos de análise e captura de lições aprendidas. Estes métodos variam enquanto a forma de registro, periodicidade, participantes, modos de iteração e benefícios. Esta seção revisa estes aspectos apresentando uma breve síntese dos métodos mais discutidos e referenciados na literatura.

\subsection{Avaliação Após Ação (After Action Review)}

A avaliação após a ação é realizada sempre que um problema é resolvido ou que qualquer ação ou decisão relevante é tomada durante o andamento do projeto. Originalmente definido pelo exército americano (Center, 1993; Schindler \& Eppler, 2003), o objetivo deste método é aprender tanto de ações 
errôneas quanto de ações exitosas imediatamente após a sua ocorrência.

A avaliação após ação tem sido proposta na literatura em diferentes formatos desde reuniões de brainstorming de 20 minutos até uma sessão de discussão de duas horas. Em comum, esses formatos sugerem a participação de toda a equipe do projeto e elementos externos que atuaram na ação relevante. A interação é realizada através de reuniões com membros da equipe de projeto de forma cooperativa coordenada por um facilitador. Neste método a equipe do projeto é confrontada com quatro questões principais (Schindler \& Eppler, 2003):

- O que era esperado acontecer?

- O que realmente aconteceu?

- Por que existiram diferenças?

- O que você pode aprender desta experiência?

Após responder estas perguntas, os membros do projeto também devem opinar sobre as ações que deverão ser tomadas de forma diferente em projetos futuros e quais membros seriam responsáveis por essas ações. As lições aprendidas são registradas pelo facilitador a fim de serem aplicadas quando necessário. De acordo com Schindler e Eppler, este método já foi usado exitosamente pela British Petroleum (BP) e pela subsidiária suíça da Siemens. $\mathrm{O}$ principal benefício desse método é permitir a equipe do projeto refletir sobre as medidas que podem ser tomadas a fim de melhorar os resultados de projetos futuros.

\subsection{Journaling}

O método denominado journaling foi proposto por Loo (2002). Um journal consiste em uma narrativa articulada que decorre do pensamento reflexivo e crítico sobre as experiências de aprendizagem ou eventos específicos de aprendizagem. Loo (2002) apresenta um modelo de aprendizagem reflexiva em três estágios: (1) consciência, a situação atual, (2) a análise crítica, que liga o presente com o passado e o futuro, e (3) a aprendizagem, o desenvolvimento de uma nova perspectiva com base na análise crítica e na aplicação de novos conhecimentos. Loo (2002) também sugere um conjunto de questões para ajudar a construir um journal:

- Qual foi a situação/evento do aprendizado?

- $\quad$ O que aprendi, e como eu aprendi isso?

- Como posso sentir (sentimentos bons ou ruins) sobre o que eu aprendi?

Como é que eu poderia ter aprendido de forma mais eficaz/eficiente?
- Que ações posso tomar para aprender de forma mais eficaz/eficiente no futuro?

- De que forma eu preciso mudar minhas atitudes, expectativas, valores e gostos para sentir-me melhor sobre as situações de aprendizagem?

Estas questões devem ser realizadas como um guia para manter o redator focado nos aspectos relevantes, entretanto podem e devem ser adicionados novos tópicos que sejam importantes para o aprendizado efetivo. Loo (2002) sugere que o journaling seja realizado pelo menos duas vezes por semana a fim de que detalhes relevantes sobre determinada situação não sejam perdidos. Através da análise crítica, o método de journaling pode ser uma ferramenta bastante útil para a equipe do projeto identificar causas raiz de problemas. Além disso, este método permite que gerentes de projetos registrem e critiquem sua própria atuação a fim de buscar a melhora contínua no seu desempenho. O journaling pode também ser utilizado como entrada para processos de coaching identificando os principais problemas e permitindo a sua priorização a fim de melhorar o desempenho de toda equipe.

\subsection{Histórias de Aprendizagem (Learning Histories)}

Histórias de aprendizagem é um método de captura de lições aprendidas proposto por Roth e Kleiner (1998). Esse método deve ser realizado após a conclusão do projeto. Uma história consiste dos principais eventos de um projeto ordenados cronologicamente. O comprimento do documento resultante pode estar entre 20 e 100 páginas seguindo a abordagem de uma história para tornar as experiências registradas mais apelativas e ricas em contexto. Essas experiências evidenciam o ponto de vista dos indivíduos envolvidos através de citações literais diretas, suprindo as deficiências de registro das lições aprendidas apresentadas por outros métodos convencionais que dificilmente expressam o conhecimento tácito. As histórias de aprendizagem geralmente iniciam com três perguntas: (1) Como podemos julgar essa experiência? (2) Como o resto da organização pode se beneficiar desta experiência? (3) Quais foram as ações que determinaram nossa falha ou sucesso? (CCS-MIT, 2014).

A aparência (layout) da página de uma história consiste normalmente em duas colunas. Os participantes das entrevistas são citados na coluna da direita e a coluna da esquerda contém comentários feitos pelos historiadores do projeto que fornecem mais contexto ou observações, especialmente no que diz respeito ao conhecimento tácito (por exemplo, interligando declarações ou descrevendo impressões sobre um dos entrevistados). Entre as colunas existem 
comentários adicionais que explicam detalhes relevantes do projeto. As caixas entre blocos de texto contêm informação sumária.

Os entrevistados são citados de forma anônima, apenas identificados para o leitor através de seus cargos (por exemplo, "gerente de projeto"), o que torna mais fácil admitir erros. Uma vez compiladas, as histórias de aprendizagem são validadas em discussões com as pessoas envolvidas. A distribuição das histórias compiladas ocorre exclusivamente via workshops (Roth \& Kleiner, 1998) ou em grupos de discussão nos quais os conteúdos são discutidos e aplicados a problemas relacionados às histórias.

$\mathrm{O}$ método historia de aprendizagem estabelece um poderoso veículo de comunicação através do processo de entrevista e durante o processo de disseminação da informação nos workshops. Pode ser utilizado como um mecanismo de transformação nas organizações permitindo que a equipe conheça suas principais debilidades, discuta e trace estratégias para superá-las.

\subsection{Micro Artigos (Micro Articles)}

Micro artigos é um método proposto por Willke (1998) para reter as experiências após a conclusão de uma atividade ou experiência relevante de um projeto. Um micro artigo é escrito num estilo informal e pode referenciar outro micro artigo. É fundamental na descrição ter o contexto do aprendizado de modo a facilitar seu reuso por leitores que não estejam familiarizados com o projeto. A dimensão de um micro artigo é limitada a meia página.

Um micro artigo consiste de um tópico, uma curta descrição introdutória sobre o seu conteúdo e as palavras-chave para sua indexação. Willke (1998) também recomenda o uso de ilustrações para suportar o aprendizado individual. Apesar de Willke (1998) recomendar o armazenamento dos micro artigos em bases de dados, deve-se considerar também o uso de tecnologias alternativas mais atuais para este processo, como por exemplo o uso de wikis ou blogs. Dado o seu formato aberto o método micro artigos permite a inclusão de objetos multimídia em seu conteúdo. A responsabilidade por redatar o micro artigo não é definida podendo ser realizada por qualquer participante do projeto. Cada experiência relevante num projeto é descrita como um micro artigo por isso esse método é descrito também como uma versão leve do método histórias de aprendizagem que agrupa todo o conhecimento de um determinado projeto em um único documento/formato. O método micro artigo também é considerado dentro do contexto de micro-learning permitindo que segmentos de informação sejam absorvidos mais efetivivamente (Gassler, Hug, \& Glahn, 2004).

\subsection{Método Apreciativo de Lições Aprendidas (Appreciative Lessons Learned Method - 4ALL)}

O método apreciativo de lições aprendidas, conhecido como 4ALL, foi definido reutilizando as melhores práticas de existentes métodos de análise e captura de lições aprendidas (Baaz et al., 2010). O objetivo deste método é ter um balanço entre excelência, desafios e mudanças gerando um melhor nível de comprometimento da equipe. Após a conclusão do projeto um moderador é designado para coordenar um workshop definindo a agenda e o foco do evento. A execução do workshop é realizada seguindo cinco premissas básicas:

- Introdução: consiste na introdução dos conceitos básicos deste método e na recapitulação do contexto do projeto. $\mathrm{O}$ moderador do workshop deve enfatizar o objetivo do evento e definir a agenda do workshop.

- Identificar excelências e desafios: o moderador do workshop deve identificar individualmente as excelências e os desafios encontrados ao longo do desenvolvimento do projeto. Esta etapa pode ser realizada no formato de uma mesa redonda na qual cada indivíduo apresenta, explica e elabora cada lição aprendida identificada.

- Organizar e decidir sobre as principais áreas: os participantes do workshop devem organizar coletivamente as lições aprendidas dentro de grandes áreas, votar nas áreas e decidir quais áreas analisar.

- Analisar e formular sugestões: o moderador do workshop deve selecionar grupos de membros do projeto com base nos interesses dos mesmos. Analisar as causas e efeitos das lições aprendidas e sugerir melhorias.

- Concordar em melhorias e concluir: o moderador do workshop deve apresentar as melhorias sugeridas, obter feedback e concordância dos outros grupos e concluir o workshop.

- Um dos principais benefícios desse método é que a análise das lições aprendidas e a definição de ações corretivas é realizada de forma colaborativa permitindo que todos participem e consequentemente tenham maior comprometimento. 


\subsection{Reuniões de Retrospectiva (Retrospective Meetings)}

Frameworks ágeis como SCRUM foram desenhados para seguimento e controle de projetos de desenvolvimento de software. Com o objetivo de prover a melhora contínua da produtividade e da qualidade dos entregáveis estes frameworks propõe reuniões de retrospectiva (Sutherland \& Schwaber, 1995). As reuniões são coordenadas pela equipe que é auto administrável com o apoio de um facilitador que coleta e organiza a informação dos demais integrantes.

As reuniões são realizadas no final de cada fase do projeto. Geralmente uma fase dura quatro semanas (Sprint). Durante as reuniões de retrospectiva a equipe analisa como foi o trabalho, se os objetivos daquela fase foram cumpridos e se as expectativas do cliente foram atendidas. Os principais pontos analisados são:

- Quais foram os aspectos positivos da última fase?

- O que foi aprendido nessa fase?

- O que é necessário melhorar para a próxima fase?

- Quais serão as próximas ações para atingir as melhoras identificadas?

- Quais são os problemas que poderiam impedir o progresso do projeto?

Após a reunião de retrospectiva o facilitador se torna responsável por apoiar a eliminar os obstáculos identificados e que não possam ser resolvidos pela própria equipe. Os principais benefícios deste método são aumento da produtividade e qualidade do produto por focarem em ações de melhora continua de curto prazo. Além disso, esse método potencializa o aprendizado da equipe de maneira sistemática e aumenta a motivação pois cada membro da equipe é visto como um agente transformador (Scrum Aliance).

\subsection{Comunidades de Prática (Communities of Practice)}

A noção de comunidades de prática $(\mathrm{CdP})$ foi desenvolvida por Lave e Wenger (1991) como uma base para uma teoria social de aprendizado. Inicialmente comunidades de prática tiveram seu o foco como uma teoria de aprendizagem e mais tarde tornaram-se parte do campo de gestão do conhecimento.

O conceito CdP consiste em um grupo de pessoas que estão conectadas informalmente que possuem um interesse comum no aprendizado teórico e prático num determinado assunto. É reconhecido que conexões significativas incrementam a criatividade e geram resultados mais rápidos do que realizados por indivíduos isolados. No contexto de gestão de conhecimento as CdPs baseiam-se na participação voluntária, numa forte relação de confiança entre os participantes e na contribuição que cada participante traz ao grupo. A organização de um CdP geralmente obedece uma estrutura não hierárquica que sugere a auto-gestão. Existe a presença de um líder que atua como facilitador promovendo a sinergia entre os participantes, organizando, capturando e disseminando o conhecimento obtido da comunidade.

Snyder e Briggs (2004) destacam os três elementos estruturais de uma comunidade de prática: o domínio, a comunidade e a prática. O domínio foca em uma área específica de assuntos relacionados (e.g. projetos de tecnologia de informação, projetos globais, gestão de risco em projetos). A comunidade inclui membros em vários níveis (e.g. gerentes, membros da equipe e demais envolvidos stakeholders). A prática constitui-se das técnicas, métodos, ferramentas e atitudes profissionais.

Atitudes profissionais envolvem atividades de aprendizagem desenvolvidas para construir, compartilhar e aplicar o conhecimento na prática. Um benefício das comunidades de prática é colmatar as fronteiras organizacionais formais, a fim de aumentar o conhecimento coletivo, as habilidades, a confiança profissional e a reciprocidade dos profissionais que atuam em organizações (Wenger, McDermott, \& Snyder, 2002).

Dentro do contexto de lições aprendidas, as comunidades de prática seria uma ação ortogonal. $\mathrm{O}$ domínio seria a gestão de projetos, a prática seria o compartilhamento de ferramentas, experiências (lições aprendidas) e instrumentos. A comunidade poderia conter todos os membros do projeto, os gestores de diferentes projetos da organização ou ainda profissionais de determinadas áreas e departamentos da empresa. Como o objetivo das comunidades de prática é prover o aprendizado contínuo a periodicidade da revisão das lições aprendidas pode variar conforme o formato do $\mathrm{CdP}$ adotado pela organização.

Entre os principais benefícios das comunidades de pratica podemos citar o ambiente favorável e confiável para intercâmbio de informação entre os participantes, utilização da experiência e conhecimento coletivo, melhora relacionamento interpessoal estabelecendo redes pessoais de longo prazo.

\subsection{Ba - Contexto Compartilhado (Ba - Shared context)}

Todo o processo de criação do conhecimento depende de um contexto específico que considere tempo, espaço e a relação com os outros. Para se 
tornar conhecimento é necessário um lugar que transforme dados em informação permitindo a interpretação e concretizando o conhecimento. Neste sentido, Nonaka et al. (2000) apresentam um modelo unificado de criação de conhecimento baseado no conceito de contexto compartilhado em movimento $(B a)$, onde o conhecimento é criado, compartilhado e utilizado. $\mathrm{O} B a$ pode emergir em indivíduos, grupos de trabalho, equipes de projeto, reuniões e espaços virtuais, entre outros. A principal ideia desse modelo é a existência de um local onde os participantes compartilham os seus contextos e criam novos significados através de suas interações. O objetivo principal da interação é estimular o $b a$. O $b a$ precisa ser "energizado" para se tornar ativo e construir significado no ambiente de trabalho (Nonaka et al., 2000). Considerando que o conhecimento precisa de um contexto físico para ser criado, $b a$ oferece tal contexto para a ação e interação. $B a$ varia de acordo com duas dimensões: tipo de interação (ou seja, individual ou coletiva) e mídia (ou seja, face-a-face ou virtual).

A principal diferença com o modelo apresentado na seção 2.7, é que nas Comunidades de Prática os grupos são estáveis e leva tempo até que um participante possa entender e contribuir efetivamente para a sua comunidade. No $B a$ o período em que um participante atua compartilhando o seu contexto é completamente variável. Um $B a$ é dado pela diversidade de participantes que contribuem com múltiplos pontos de vista e assim compartilham de contextos variados.

Dentro do contexto de lições aprendidas, o $B a$ pode ser aplicado através da interação de membros de diferentes projetos a fim de compartilhar diferentes experiências e visões distintas sobre a mesma experiência. Neste modelo a periodicidade e os participantes podem variar bastante. Esse modelo conta com a presença de um líder, que sintetiza o conhecimento adquirido através dos Bas em que participa. O principal benefício do $B a$ é proporcionar condições favoráveis a uma sinergia ativa aos processos de criação e ampliação de conhecimento. Como restrição deste modelo é necessário mencionar que ainda parece se tratar de um conceito filosófico com pouca aplicação prática.

\subsection{Avaliação do Projeto (Project Review) e Avaliação Pós-Projeto (Postcontrol ou Post-Project Review)}

O método de avaliação do projeto pode ser realizado após a finalização do mesmo ou durante suas fases individuais. Este método é aplicado por meio de reuniões presenciais e conta com moderadores (membros do projeto) e auditores (pessoas externas ao projeto). O propósito deste método que possui um foco na equipe interna do projeto é identificar previamente os possíveis riscos do projeto. Nesse método inicialmente é descrito o contexto em que se aplica o projeto. A seguir são identificadas e detalhadas as principais técnicas, abordagens, decisões que foram importantes para o êxito do projeto a fim de que possam ser reutilizadas em projetos futuros. Também são identificados ações, técnicas e abordagens que geraram problemas a fim de que sejam divulgados como má praticas em projetos.

Como o título sugere, a avaliação pósprojeto é realizada no fim do projeto pelo gerente e com a participação de toda a equipe do projeto. $\mathrm{O}$ propósito é formalizar a finalização do projeto e concentrar nas lições aprendidas a fim de identificar ações que permitam melhorar o desempenho de toda a equipe nos projetos futuros. A interação é feita de forma não-cooperativa através do registro de experiências, da análise dos relatórios de status existentes, atas de reunião e qualquer documentação relevante do projeto. $\mathrm{O}$ resultado é um documento formal que considera o escopo do projeto, os objetivos quantitativos e qualitativos, marcos de controle, metas orçamentárias, avaliação do projeto e recomendação de ações para futuras melhorias. Os principais benefícios dessas duas abordagens incluem a melhoria da disciplina da equipe, a prevenção dos pontos fracos e a validação das estratégias utilizadas no projeto.

\subsection{Apreciação Pós-Projeto (Post-Project Appraisal)}

O método de apreciação pós-projeto foi proposto por Gulliver (1987) e utilizado por empresas como a British Petroleum e a Boing. Esse método é geralmente usado após dois anos de conclusão de projetos de grandes dimensões. Segundo Gulliver, a justificativa para aplicar esse método em projetos desta envergadura é que as lições mais valiosas são obtidas de projetos que apresentam maior custo, numero de pessoas e diversidade de perfis.

Neste método, cria-se uma unidade de apreciação pós-projeto na organização. Essa unidade é formada por um conjunto de profissionais responsáveis pela realização da avaliação do projeto. O objetivo é ter uma equipe externa ao projeto que realize uma analise objetiva e evitar possíveis influências dos membros do projeto na avaliação. Essa equipe avalia toda documentação do projeto $\mathrm{e}$ realiza entrevistas. Em média 40 profissionais são entrevistados num processo que leva cerca de seis meses.

O relatório resultante da aplicação deste método é submetido aos membros da equipe do projeto e revisado por um conselho de avaliação antes de ser oficialmente lançado. Uma vez aprovado, o resultado é disponibilizado como uma coleção de 
casos que serão usados em projetos futuros. Gulliver (1987) descreve que essa disponibilização seja feita em papel, mas dado o contexto atual das organizações essa disponibilização pode ser feita em formato digital. Os maiores benefícios da aplicação deste método são: identificação de melhores práticas, melhora significativa na estimação, projeção e propostas de projetos grandes dimensões.

\subsection{Documentação Leve de Experiências (Light- weight Documentation of Experiences - LIDs)}

O método LID procura capturar as experiências obtidas logo após a conclusão de uma atividade em uma organização. As pessoas envolvidas na atividade são contatadas para contar as experiências obtidas por meio de uma história. A documentação coletada tanto na história quanto nos templates existentes para execução da tarefa deve ser coloca num local comum (e.g. uma pasta de arquivos) para facilitar o acesso subsequente das pessoas interessadas no aprendizado obtido na atividade (Schneider, 2000). De acordo com Schneider (2000), a abordagem LID pode ser descrita genericamente como segue:

1. Pouco depois de uma atividade acabar, faça com que alguns participantes-chave contém a história. Use uma breve lista de perguntas e coisas que você quer aprender.

2. Escreva a história junto com eles em algumas páginas, e recolha todas as versões mais recentes dos documentos mencionados na história (por exemplo, slides, planos e relatórios) que tiveram alguma relevância.

3. Em particular, procure modelos (templates) de documentos.

4. Coloque tudo isso em um diretório (como em um pote), vincule a história aos documentos a que ela se refere e use a história como o (único) mecanismo de acesso para os ingredientes no pote (a tampa - lid - cobre o pote). Por tampa, entende-se um mecanismo de acesso restritivo.

O método LID foi usado no Centro de Experiência em Software (Software Experience Center - SEC) da empresa automobilística alemã DaimlerChrysler. Este método é adequado para situações específicas tais como a introdução de novas tecnologias em um pequeno grupo de trabalho, o treinamento de uma atividade de um projeto como a escrita de planos e casos de teste, e a organização e realização de workshops ou em uma reunião de especialistas.

\section{ANÁLISE DOS MÉTODOS PARA APRENDIZADO EM PROJETOS}

Esta seção apresenta uma análise dos principais métodos para aprendizado em projetos. As Figuras 1, 2 e 3 sintetizam uma análise dos principais critérios para avaliação dos métodos para aprendizado em projetos. $\mathrm{O}$ critério aplicabilidade procura identificar a fase do projeto mais indicada para o método ser usado. Os participantes são as pessoas envolvidas no projeto que devem participar do processo de captura de lições aprendidas. A duração estimada é uma estimativa superficial do tempo total a ser investido na implementação do método. Deve-se considerar a dimensão do projeto para se estabelecer uma estimativa mais precisa. $\mathrm{O}$ valor do critério das organizações que usam ou já usaram o método é proveniente dos artigos nos quais os métodos estão descritos bem como de referências encontradas na Web. A tecnologia de suporte é uma sugestão de tecnologias que podem suportar a implementação do método. A data da primeira publicação apresenta o ano no qual o método foi descrito pela primeira vez na literatura. Finalmente, o critério referência cita a principal referência que descreve o método. Os participantes dos métodos de captura de lições aprendidas são o Gerente de Projeto, a sua equipe e os stakeholders, sendo que em alguns métodos esses últimos não participam. As tecnologias indicadas para suportar o aprendizado que emergirá da aplicação dos métodos estão em linha com o gerenciamento de projetos moderno ou gerenciamento de projetos 2.0 (Levitt, 2011). Essas tecnologias estão presentes na Web 2.0 e permitem a implementação de uma dinâmica colaborativa durante o uso do método. Os exemplos de organizações que usaram os métodos evidenciam que o aprendizado em projetos é uma preocupação, independentemente da dimensão da organização. Grandes organizações são listadas nas Figuras 1, 2 e 3. Von Zedtwitz (2002) descreve o uso de avaliação pós-projeto (AvPP) em projetos de pesquisa e desenvolvimento em grandes organizações. No Reino Unido, Suresh (2006) conduziu um estudo empírico com 26 pequenas e médias empresas da indústria da construção, que aplicaram o método de análise pós projeto (AAA) informalmente. Carrillo e Anumba (2002) relatam que comunidades de prática é o método mais usado para gestão do conhecimento em grandes organizações do setor da construção também no Reino Unido. Probst e Borzillo (2008) apresentam um estudo com mais de 20 organizações de diversos setores que utilizaram comunidades de prática. Por outro lado, as reuniões de retrospectiva são comumente usadas em domínios específicos como os projetos de Tecnologias de Informação que envolvem desenvolvimento ágil, independente da dimensão da organização. 


\begin{tabular}{|c|c|c|c|c|}
\hline CRITÉRIO & $\begin{array}{l}\text { AVALIAÇÃO DO } \\
\text { PROJETO }\end{array}$ & $\begin{array}{c}\text { AVALIAÇÃO PÓS- } \\
\text { PROJETO }\end{array}$ & $\begin{array}{l}\text { APRECIAÇÃO } \\
\text { PÓS PROJETO }\end{array}$ & $\begin{array}{l}\text { COMUNIDADES DE } \\
\text { PRÁTICA }\end{array}$ \\
\hline Aplicabilidade & Durante o projeto & No fim do projeto & $\begin{array}{l}2 \text { anos após o fim do } \\
\text { projeto }\end{array}$ & Durante o projeto \\
\hline Participantes & $\begin{array}{l}\text { GP, equipe e } \\
\text { stakeholders }\end{array}$ & $\begin{array}{l}\text { GP, equipe e } \\
\text { stakeholders }\end{array}$ & $\begin{array}{l}\text { GP, equipe e } \\
\text { stakeholders }\end{array}$ & $\begin{array}{c}\text { GP, facilitador, equipe e } \\
\text { stakeholders }\end{array}$ \\
\hline Duração estimada & Variável & Variável & 6 meses & Variável \\
\hline $\begin{array}{c}\text { Exemplos de } \\
\text { organizações que } \\
\text { usam ou já usaram }\end{array}$ & $\begin{array}{l}\text { Kennedy Space } \\
\text { Center }\end{array}$ & $\begin{array}{c}\text { Hewlett-Packard, } \\
\text { DaimlerChrysler, SAP } \\
\text { e Unisys }\end{array}$ & BP e Boing & $\begin{array}{l}\text { Siemens, Oracle, IBM, } \\
\text { CERN e Pioneer }\end{array}$ \\
\hline $\begin{array}{l}\text { Tecnologia como } \\
\text { suporte }\end{array}$ & Blogue, VoIP e Wiki & Blogue, VoIP e Wiki & Blogue, VoIP e Wiki & Wiki e Office \\
\hline $\begin{array}{l}\text { Data da } 1^{\mathrm{a}} \\
\text { publicação }\end{array}$ & 1977 & 1977 & 1987 & 1991 \\
\hline Referência & Faas (1977) & Skeen (1977) & Gulliver (1987) & Lave e Wenger (1991) \\
\hline
\end{tabular}

Figura 1 - Métodos para aprendizado em projetos. AP: Avaliação do Projeto; AvPP: Avaliação Pós-Projeto; ApPP: Apreciação Pós-Projeto; CdP: Comunidades de Prática.

Fonte: Elaborado pelos autores.

\begin{tabular}{|c|c|c|c|c|}
\hline CRITÉRIO & $\begin{array}{l}\text { ANÁLISE PÓS } \\
\text { PROJETO }\end{array}$ & $\begin{array}{c}\text { REUNIÕES DE } \\
\text { RETROSPECTIVA }\end{array}$ & $\begin{array}{c}\text { MICRO } \\
\text { ARTIGOS }\end{array}$ & $\begin{array}{c}\text { HISTÓRIAS DE } \\
\text { APRENDIZAGEM }\end{array}$ \\
\hline Aplicabilidade & Durante o projeto & No fim de cada sprint & Durante o projeto & Durante o projeto \\
\hline Participantes & $\begin{array}{l}\text { GP, facilitador e } \\
\text { equipe }\end{array}$ & $\begin{array}{l}\text { GP, facilitador e } \\
\text { equipe }\end{array}$ & GP e equipe & GP e equipe \\
\hline Duração estimada & Entre 20 min. e 2 horas & 3 horas & Máximo 1 turno & Vários dias \\
\hline $\begin{array}{c}\text { Exemplos de } \\
\text { organizações que } \\
\text { usam ou já usaram }\end{array}$ & $\begin{array}{l}\text { BP, Siemens e Forças } \\
\text { Armadas Americanas. }\end{array}$ & $\begin{array}{l}\text { Google, Stefanini IT } \\
\text { Solutions, CI\&T }\end{array}$ & $\begin{array}{l}\text { Não encontrado na } \\
\text { literatura }\end{array}$ & $\begin{array}{c}\text { Sloan School of Management } \\
\text { at MIT }\end{array}$ \\
\hline $\begin{array}{l}\text { Tecnologia como } \\
\text { suporte }\end{array}$ & Wiki, blogue e Office & Wiki e Office & Blogue ou Wiki & Office \\
\hline $\begin{array}{l}\text { Data da } 1^{\mathrm{a}} \\
\text { publicação }\end{array}$ & 1993 & 1995 & 1998 & 1998 \\
\hline Referência & Center (1993) & $\begin{array}{c}\text { Sutherland e Schwaber } \\
\text { (1995) }\end{array}$ & Willke (1998) & Roth e Kleiner (1998) \\
\hline
\end{tabular}

Figura 2 - Métodos para aprendizado em projetos (continuação). AAA: Análise Pós-Projeto; RR: Reuniões de Retrospectiva; MA: Micro Artigos; HA: Histórias de Aprendizagem.

Fonte: Elaborado pelos autores. 


\begin{tabular}{|c|c|c|c|c|}
\hline CRITÉRIO & $\begin{array}{c}\text { DOCUMENTAÇÃO } \\
\text { LEVE DE } \\
\text { EXPERIENCIAS }\end{array}$ & $\begin{array}{c}\text { CONTEXTO } \\
\text { COMPARTILHADO }\end{array}$ & JOURNALING & 4ALL \\
\hline Aplicabilidade & $\begin{array}{c}\text { Após uma curta } \\
\text { atividade (de 1 a 3 } \\
\text { meses) }\end{array}$ & Durante o projeto & $\begin{array}{c}\text { Duas vezes por } \\
\text { semana }\end{array}$ & $\begin{array}{c}\text { Após a conclusão do } \\
\text { projeto }\end{array}$ \\
\hline Participantes & GP e equipe & GP, facilitador e equipe & GP e equipe & $\begin{array}{c}\text { GP, moderador, equipe e } \\
\text { stakeholders }\end{array}$ \\
\hline Duração estimada & Máximo 2 horas & Variável & Máximo 1 turno & 1 ou 2 turnos \\
\hline $\begin{array}{c}\text { Exemplos de } \\
\text { organizações que usam } \\
\text { ou já usaram }\end{array}$ & DaimlerChrysler & $\begin{array}{c}\text { Não encontrado na } \\
\text { literatura }\end{array}$ & $\begin{array}{c}\text { Lethbridge (alunos } \\
\text { de graduação em } \\
\text { Administração) }\end{array}$ & Ericsson \\
\hline $\begin{array}{c}\text { Tecnologia como } \\
\text { suporte }\end{array}$ & Wiki & $\begin{array}{c}\text { Wiki, blogue, Office e } \\
\text { VoIP }\end{array}$ & $\begin{array}{c}\text { Wiki, blogue e } \\
\text { Office }\end{array}$ & Wiki \\
\hline Data da 1 ${ }^{\text {a publicação }}$ & 2000 & 2000 & 2002 & Baaz et al. (2010) \\
\hline Referência & Schneider (2000) & Nonaka, et al. (2000) & Loo (2002) & \\
\hline
\end{tabular}

Figura 3 - Métodos para aprendizado em projetos (continuação). LID: Documentação Leve de Experiências; Ba: Contexto Compartilhado; J: Journaling; 4ALL: Método Apreciativo de Lições Aprendidas.

Fonte: Elaborado pelos autores.

\section{DISCUSSÃO}

Os projetos são utilizados pelas organizações para produção e desenvolvimento de bens e serviços e apresentam inúmeros desafios durante o seu ciclo de vida que incluem problemas contratuais, estimativas erradas, baixo desempenho da equipe, falta de conhecimento dos provedores e substituição de profissionais. Identificar cada desafio e agir proativamente buscando soluções para cada situação que se presente, é crucial para o término exitoso dos projetos. Desta forma, o uso de métodos que permitam documentar e distribuir o aprendizado obtido em cada uma dessas experiências é uma ferramenta inestimável para a organização, permitindo reduzir custos, reduzir riscos, aumentar produtividade e competividade.

Com objetivo de maximizar os resultados dos projetos, as organizações têm adotado amplamente os métodos e técnicas difundidos pelo Project Management Institute (PMI). O PMI é uma instituição internacional que investiga e promove as melhores práticas de gestão de projetos. Entretanto, o tópico de lições aprendidas tem sido subvalorizado no guia PMBoK. O guia descreve que as lições aprendidas devem ser capturadas como informação histórica no fim de cada fase ou no encerramento do projeto. Entretanto, o uso de lições aprendidas prevê o estudo e a implantação de uma metodologia de transferência de conhecimento e não se limita apenas a um conjunto de atividades executadas durante uma determinada fase do projeto. Senge (1994) descreve organizações de aprendizagem como organizações em que as pessoas continuamente expandem seus conhecimentos para criar os resultados que verdadeiramente desejam. Novos padrões de pensamento são nutridos, a aspiração coletiva é libertada e as pessoas passam a continuamente aprender a aprender. A utilização de métodos de captura de lições aprendidas está dentro deste contexto, permitindo que a evolução através da experiência obtida com um projeto seja um aprendizado proposital e não casual. Considerando os métodos de captura de lições aprendidas descritos neste artigo, as organizações podem incluir o aprendizado como um objetivo explícito do projeto. $\mathrm{O}$ uso de métodos de captura de lições aprendidas permite que o aprendizado no projeto seja proposital e deliberado, ao invés de acidental e oportunista.

Alguns métodos de captura de lições aprendidas, apresentados nesse artigo, propõem que a análise e registro das experiências vivenciadas no projeto sejam realizadas após a conclusão do mesmo (ver Figuras 1, 2 e 3). Para maximizar o uso do conhecimento obtido em determinada experiência, se faz necessário que toda informação relevante seja documentada logo após a sua ocorrência (Anbari, 1985). Realizar o registro apenas ao final do projeto permite que se perca detalhes importantes dos acontecimentos. Devido ao caráter dinâmico das organizações, profissionais se movem para outras áreas, levando consigo conhecimento de ações e decisões que foram fundamentais para o projeto. Quando o registro das experiências ocorre de forma 
continua, durante todo o ciclo de vida do projeto, cria-se uma disciplina na equipe permitindo que essa revise seu próprio desempenho, reflexione sobre as ações exitosas e definam ações corretivas de curto prazo. Sendo assim, o registro contínuo beneficia o projeto per se e projetos futuros.

A definição de como serão registradas as lições aprendidas permanece um dos grandes desafios para a implantação de métodos de captura. Formatos extensos, que considerem registrar a visão de cada um dos participantes como sugerido pelos métodos de Apreciação Pós-Projeto e Histórias de Aprendizagem podem ser custosos e não viáveis para muitas empresas. Ao selecionar o formato dos registros das lições aprendidas, deve-se ter em mente como esse conteúdo será apresentado e distribuído a fim de que o acesso a essa informação seja simples, dinâmico e atinja o maior número de profissionais. Atualmente várias tecnologias modernas podem facilitar a captura e a disseminação das lições aprendidas, conforme apresentado nas Figuras 1, 2 e 3. Cleveland (2012) propõe o uso de microblogues na tarefa de registro de lições aprendidas em projetos. Parker e Chao (2007) enfatizam o uso de wikis para melhorar o processo de aprendizagem em organizações. Hsu e Lin (2008) verificaram que a facilidade de uso, o aproveitamento (enjoyment), e o compartilhamento de conhecimento (altruísmo e reputação) estavam positivamente relacionados à atitude em relação aos blogues. Deve-se também considerar que algumas dessas tecnologias podem ser usadas em reuniões nos projetos.

As reuniões para apresentar e discutir as lições aprendidas são cruciais para a concretização da transferência de conhecimento. As reuniões podem diferir no formato, periodicidade e na duração dependendo do método. Seja qual for o método utilizado a garantia de um ambiente colaborativo e de aprendizado em que todos os participantes se sintam como colaboradores de um processo de transformação é fundamental. Para promover esse ambiente, a presença de um facilitador é sugerida em vários métodos apresentados. $\mathrm{O}$ facilitador deve ter uma posição neutra e orientar o grupo para a realização de uma análise objetiva e definição de ações corretivas.

Os métodos de Reuniões de Retrospectiva (RR) e Apreciativo (4ALL) definem um framework para realização das reuniões e acompanhamento das ações corretivas (pós-aprendizado). O facilitador acompanha e remove impedimentos, apoiando toda a equipe na implantação das mudanças necessárias. $\mathrm{O}$ acompanhamento das ações corretivas é relevante para que todos os participantes percebam a evolução e o comprometimento dos envolvidos.

O principal objetivo dessa seção foi apresentar e discutir os aspectos que devem ser levados em consideração na avaliação de métodos para aprendizados em projetos. Cada método possui características específicas que podem ser combinadas a fim de melhor adaptar a metodologia de transferência de conhecimento ao ambiente organizacional, tipo ou tempo médio de execução dos projetos. É importante ressaltar, que a implantação de métodos de aprendizado somente é exitosa dentro de uma organização se há o envolvimento da alta administração da empresa. Esforços individuais não são eficientes e eficazes se a gestão de conhecimento não é um aspecto cultural da organização. Se a alta administração não é sensibilizada da importância do processo, as atividades diárias tomam prioridade sobre o registro, captura e discussão das lições aprendidas, tornando os resultados contraproducentes.

Por fim, deve-se referir que uma das limitações deste trabalho é número de critérios usados na análise dos métodos de captura de lições aprendidas, os quais precisam ser estendidos para permitir uma análise mais profunda sobre o aprendizado em projetos. Alguns dos aspectos a considerar nos métodos e atividades para aprendizado incluem o grau de formalidade (i.e. formal e informal), se são públicos ou privados, e se podem ser realizados virtualmente ou de forma presencial.

\section{RECOMENDAÇÕES E CONCLUSÃO}

Essa seção conclui o artigo com os principais pontos que devem ser considerados na escolha e implantação dos métodos de aprendizado dentro do contexto de gestão de projetos:

- Ambiente organizacional: os desafios de um ambiente projetizado são diferentes de uma organização matricial. Nos ambientes orientados a projetos é mais fácil integrar objetivos de aprendizado dentro dos objetivos do projeto. Ambientes matriciais devem considerar os objetivos de aprendizado entre diferentes áreas da organização.

- Definição de objetivos tangíveis: a definição e o acompanhamento de objetivos, durante $\mathrm{o}$ processo de implantação e execução dos métodos de aprendizado, tornam possível avaliar os benefícios e continuamente sensibilizar stackeholders e diretores sobre a importância dos processos de transferência de conhecimento.

- Análise do tempo médio de execução dos projetos: projetos de curto prazo (3 a 6 meses) devem utilizar métodos mais dinâmicos como Micro Artigo e Reuniões de Retrospectiva. Para projetos de médio a largo prazo podem ser utilizados métodos 
com formatos detalhados e extensos como Histórias de Aprendizagem e Avaliação do Projeto.

- Revisão continua das lições aprendidas: evita perda de conhecimento e promove equipes disciplinadas, análise de desempenho, reflexão de ações exitosas, e definição de ações corretivas.

- Captura dinâmica: os métodos e formatos de captura devem ser dinâmicos e de fácil acesso fazendo com que os participantes se sintam motivados a compartilhar suas experiências. Atualmente, um conjunto de tecnologias denominadas de Web 2.0 estão complementando e transformando a gestão de projetos através de ferramentas colaborativas e que permitem disseminar a informação em diferentes mídias sociais (e.g. vídeo, voz, redes sociais e grupos de discussão).

- Reuniões de retrospectiva com facilitador: com o treinamento adequado, o facilitador permite o direcionamento dos participantes tornando as reuniões mais ágeis e assegurando um ambiente colaborativo.

- Seguimento e controle das ações: a fim de motivar os participantes é importante que todos identifiquem os resultados das ações definidas nas reuniões de retrospectiva permitindo que cada participante se sinta como um agente transformador.

- Métricas: com base na informação proveniente da captura das lições aprendidas, organizações devem ser capazes de periodicamente transformar essas informações em indicadores de qualidade e maturidade da gestão de projetos.

Adicionalmente, a gestão de lições aprendidas em projetos pode combinar o uso de diversos métodos de captura e análise. Métodos que podem ser usados durante todo o projeto podem ser combinados com métodos usados exclusivamente após a conclusão do projeto. Por exemplo, a opção de uso do método LID ao longo do projeto e do método 4ALL com um workshop após a conclusão do projeto pode ser uma alternativa interessante para reter e disseminar o conhecimento.

Em resumo, este artigo descreveu os principais métodos de captura de lições aprendidas encontrados na literatura e discutiu recomendações de uso destes métodos em gestão de projetos. Dada a escassez de artigos em português que apresentem e discutam os métodos captura e lições aprendidas, esta pesquisa também contribui para um melhor entendimento dos métodos descritos por parte de pesquisadores que estão iniciando na área. Neste sentido, este trabalho também propõe uma terminologia em português, suprindo uma carência da literatura nesse tópico. Finalmente, a análise da aplicação de métodos para captura de lições aprendidas em empresas no Brasil ainda é uma questão em aberto, o que gera a necessidade de realização de estudos de caso exploratórios.

\section{REFERÊNCIAS}

Anbari, F.T. (1985). A systems approach to project evaluation. Project Management Journal 16(3), 21-26.

Anbari, F. T., Carayannis, E. G., \& Voetsch, R. J. (2008). Post-project reviews as a key project management competence. Technovation, 28(10), 633-643.

Baaz, A., Holmberg, L., Nilsson, A., Olsson, H. H., \& Sandberg, A. B. (2010). Appreciating Lessons Learned. Software, IEEE, 27(4), 72-79.

Busby, J.S. (1999). An assessment of post-project reviews. Project Management Journal, 30 (3), 23-29.

Carrillo, P.M. and Anumba, C.J. (2002). Knowledge management in the AEC sector: an exploration of the mergers and acquisition context. Knowledge and Process Management, 9(3), 149-161.

CCS-MIT (2014). Center for Coordination Science at MIT Sloan. Avaiable at http://ccs.mit.edu/lh/intro.html. Last visit $\underline{04 / 22 / 2014}$.

Center, U. A. C. A. (1993). A leader's guide to afteraction reviews (Training Circular 25-20). Fort Leavenworth, $K S$.

Cheah, Y., Khoh, S. B., \& Ooi, G. B. (2011). An ontological approach for Program Management Lessons Learned: Case study at Motorola Penang Design Centre. In Industrial Engineering and Engineering Management (IEEM), IEEE International Conference on, 1612-1616.

Cleveland, S. (2012). Using Microblogging for Lessons Learned in Information Systems Projects. In International Research Workshop on IT Project Management (pp. 122-128).

Collier, B., DeMarco, T., \& Fearey, P., (1996). A defined process for project postmort em review. IEEE Software Journal, 65-71. 
Faas, R. C. (1977). Decision making in project review: processes, participation, and performance in Michigan multi county comprehensive health planning. Agric Econ Rep Dep Agric Econ Mich State Univ.

Ferenhof, H. A., Forcellini, F. A., \& Varvakis, G. (2013). Lições Aprendidas: Agregando Valor ao Gerenciamento de Projetos. Revista Gestão $e$ Projetos, 4(3), 197-209.

Gassler, G., Hug, T., \& Glahn, C. (2004). Integrated Micro Learning - An outline of the basic method and first results. Interactive Computer Aided Learning, 4.

Gulliver, F. R. (1987). Post-project appraisals pay. Harvard Business Review, 65(2), 128.

Hobday, M. (2000). The project-based organisation: An ideal form for managing complex products or systems?. Research Policy, 29(7-8), 871-893.

Hsu, C.-L., \& Lin, J. C.-C. (2008). Acceptance of blog usage: The roles of technology acceptance, social influence and knowledge sharing motivation. Information \& Management, 45(1), 65-74.

Jugdev, K. (2012). Learning from Lessons Learned: Project Management Research Program. American Journal of Economics and Business Administration, 4(1), 13-22.

Kotnour, T., \& Vergopia, C. (2005). Learning-Based Project Reviews: Observations and Lessons Learned from the Kennedy Space Center. Engineering Management Journal, 17(4).

Lave, J. \& Wenger, E. (1991). Situated Learning: Legitimate Peripheral Participation. Cambridge: Cambridge University Press. ISBN 0-521-423740 .

Levitt, R. E. (2011). Towards project management 2.0. Engineering Project Organization Journal, 1(3), 197-210.

Loo, R. (2002). Journaling: A learning tool for project management training and team-building. Project Management Journal, 33(4), 61-66.

Nonaka, I., Toyama, R., \& Konno, N. (2000). SECI, $B a$ and Leadership: a Unified Model of Dynamic Knowledge Creation. Long Range Planning, 33(1), 5-34.
Parker, K., \& Chao, J. (2007). Wiki as a Teaching Tool. Interdisciplinary Journal of E-Learning and Learning Objects (IJELLO), 3(1), 57-72.

Probst, G., \& Borzillo, S. (2008). Why communities of practice succeed and why they fail. European Management Journal, 26(5), 335-347.

Roth, G., \& Kleiner, A. (1998). Developing organizational memory through learning histories. Organizational Dynamics, 27(2), 43-60.

Schindler, M., \& Eppler, M. J. (2003). Harvesting project knowledge: a review of project learning methods and success factors. International Journal of Project Management, 21(3), 219-228.

Schneider, K. (2000). LIDs: a light-weight approach to experience elicitation and reuse. In Product Focused Software Process Improvement (pp. 407424). Springer Berlin Heidelberg.

Senge, P. M. (1991). The fifth discipline, the art and practice of the learning organization. Doubleday, NY, First Edition, pp: 412.

Sense, A. J. (2004). An architecture for learning in projects? Journal of Workplace Learning, 16(3), 123-145.

Sense, A. J. (2007). Cultivating learning within projects. Palgrave Macmillan, London, pp: 220.

Sense, A. J. (2008). Conceptions of learning and managing the flow of knowledge in the projectbased environment. International Journal of Managing Projects in Business, 1(1), 33-48.

Skeen, D. R. (1977). User involvement with EDP systems development. In Proceedings of the fifteenth annual SIGCPR conference (pp. 62-71). ACM.

Snyder, W. M., \& Briggs, X. de S. (2004). Communities of practice: A new tool for government managers. Collaboration: Using networks and partnerships, 171-272.

Suresh S. (2006). Knowledge management in small and medium enterprises in the United Kingdom construction industry, $\mathrm{PhD}$ thesis, Glasgow Caledonian University, Scotland, UK.

Sutherland, J. V. \& Schwaber, K. (1995). Business object design and implementation: OOPSLA '95 workshop proceedings. The University of Michigan. p. 118. ISBN 3-540-76096-2. 
Swan, J., Scarbrough, H., \& Newell, S. (2010). Why don't (or do) organizations learn from projects? Management Learning, 41(3), 325-344.Weber, R., Aha, D. W., \& Becerra-Fernandez, I. (2001). Intelligent lessons learned systems. Expert Systems with Applications, 20(1), 17-34.

Von Zedtwitz, M. (2002). Organizational learning through post-project reviews in R\&D. $R \& D$ Management, 32(3), 255-268.
Wenger, E., McDermott, R., \& Snyder, W. M. (2002). Cultivating Communities of Practice: A Guide to Managing Knowledge (Boston: Harvard Business School Press).

Williams, T. (2008). How do organizations learn lessons from projects - And do they? Engineering Management, IEEE Transactions on, 55(2), 248266.

Willke, H. (1998). Systemisches Wissensmanagement. Stuttgart: Lucius \& Lucius Verlagsgesellschaft. 